\title{
EDITORIAL
}

\section{Broadening Design and Designers’ Perspective}

\author{
Serena Graziosi ${ }^{a *}$, Armando Viviano Razionale ${ }^{b}$ \\ ${ }^{a}$ Department of Mechanical Engineering, Politecnico di Milano, Milan, Italy \\ ${ }^{b}$ Department of Civil and Industrial Engineering, Università di Pisa, Pisa, Italy
}

The rapid diffusion of advanced hardware and software technologies and the societal challenges (Castelo-Branco et al., 2019; Martin and Leurent, 2017; United Nations, 2015), which we are urgently asked to face now and in the next years, are demanding for a widening of our design perspective. Design activities are becoming even more multidisciplinary, and designers are requested to properly evaluate in advance the effects of their solutions and the potential consequences of their decisions at a broad spectrum. Such an increase in the complexity of design activities should be seen as a stimulus and as a natural evolution of all those design processes intended to introduce radical and positive changes in our daily life. However, to properly tackle this challenge designers should continuously update their professional and cultural knowledge and skills (Dym et al., 2005) while hardware and software tools should be made, even more, widely accessible to all (e.g., see (Von Hippel, 2005)). Indeed, researchers should concentrate their efforts on developing technologies for guiding designers to take up such complexity properly, and on improving dedicated design methods, tools and guidelines. They should help designers to exploit the design potentials of current and next hardware and software technologies, and push designers to deal with such complexity more systematically.

Additive Manufacturing (AM) technologies are an example of technologies that have now reached a high level of accessibility thanks to their wide diffusion. They have been conceived to enlarge the solutions space by providing more design freedom (Thompson et al., 2016): they enable the exploration of advanced design possibilities, for example, by combining multiple materials and by working at different manufacturing scales. Alone or in combination with others, they thus represent an enabling technology for the development of innovative products/services. However, considering their rapid evolution and the advanced and multidisciplinary design scenario they are offering, designers should be trained on how to, successfully, Design for Additive Manufacturing (DfAM) (Rosen et al., 2015); indeed, design process/activities can significantly benefit from the potentialities and the advantages that these technologies can provide.

The papers of this issue demonstrate the fundamental role played by AM technologies, on the one hand, and the implementation of structured and comprehensive design strategies, on the other, in broadening design and designers' perspective. Besides, it is the proper combination of these two aspects that can lead to significant steps forward when designing new products/services.

The first paper, “3D printed 3D-Microfluidics: recent developments and design challenges” provides a useful state of the art in the field of the fabrication of 3D-Microfluidics devices using AM technologies. In this paper, it is shown how the technological improvement in AM could lead to a new approach in the design of microfluidic devices by allowing for intricate 3D channels that could lead to innovative solutions and application. Examples of new possibilities in the design and manufacturing of 3D

\footnotetext{
${ }^{*}$ Corresponding author. Email: serena.graziosi@polimi.it
} 
Microelectromechanical Systems (MEMS) and 3D-Microfluidic devices are described. The paper offers an overview of the specific challenges to be addressed from the designer for the direct 3D printing of such devices and an analysis of the key parameters (i.e., design, manufacturing and process parameters) to be considered in order to obtain affordable and reliable new devices. The research in this field, thus, is pushed ahead thanks to the developed new technologies and the ability of the designer to deeply know their potentialities and capabilities.

The second paper, "a co-design method for the additive manufacturing of customised assistive devices for hand pathologies", demonstrates how the direct involvement of the end-user (in this case the patient) in a product customisation process, enabled by AM technologies, is a winning approach. This involvement is fundamental for guaranteeing tailored solutions able to fulfil end-users' needs fruitfully; these needs are multifaceted since they are not only related to functional aspects but also involved with psychological and ethical ones. Besides, a systematic design approach, based on parametric virtual models, is also discussed in this paper. The parametric model provides a quick and efficient exploration of the design space and acts as a reference for the multidisciplinary team involved in the design process. In this paper, it is through the effective combination of technological and methodological aspects that design targets are successfully fulfilled.

The third paper, "a system design reference model-based on design processes and designers' experience", describes a reference model to be used as a tool for identifying commonalities, distinguishing features and complementarities among the vast number of design methods available in the literature. Three scales are used to map them by considering the level of complexity of the design problem managed, how the problem is approached (whether at an abstract or a concrete level) and at which moment of the design process or, of the system life-cycle, the selected design method works. Indeed, one of the main purposes of this mapping is to help designers to identify the most suitable design approach, or the appropriate combination of different design approaches, with respect to the design challenge to be tackled. This paper is thus focused on the methodological aspect; it highlights how the design of complex systems also requires a reasoned and informed selection of the adequate design tools and approaches, to be used/implemented.

The papers of this special issue show that designers are already aware of the new opportunities provided by the continuous development of software and hardware tools. However, they should be supported in deepening their knowledge about these tools in order to broaden their perspective and successfully catch these opportunities. Further research efforts, both theoretical and practical, are needed to guide designers on how to deal with complex and multidisciplinary design activities. Case studies and discussions involving researchers from multiple disciplines and working on a common design challenge, would be of great relevance to enrich the background knowledge on this topic. Besides, studies explaining and/or providing examples on how to train next generation of designers in enlarging their perspective, are also of great relevance to this aim. Finally, further contributions are also expected on how the design activity can take advantages of the potentials of new hardware and software technologies both in the design of new products/services as well as in making more efficient the design process.

\section{References}

Castelo-Branco, I., Cruz-Jesus, F., and Oliveira, T. (2019), “Assessing Industry 4.0 readiness in manufacturing: Evidence for the European Union”, Computers in Industry, Vol. 107, pp. 22-32, https://doi.org/10.1016/j.compind.2019.01.007.

Dym, C. L., Agogino, A. M., Eris, O., Frey, D. D., and Leifer, L. J. (2005), “Engineering design thinking, teaching, and learning”, Journal of engineering education, Vol. 94(1), pp. 103-120, https://doi.org/10.1002/j.2168-9830.2005.tb00832.x.

Martin, C., and Leurent, H. (2017), "Technology and innovation for the future of production: Accelerating value creation”, World Economic Forum, available at www.weforum.org.

Rosen, D. W., Seepersad, C. C., Simpson, T. W. and Williams, C. B. (2015), "Special issue: Design for additive manufacturing: A paradigm shift in design, fabrication, and qualification”, Journal of Mechanical Design, Vol. 137(11), 110301-110301-2, http://dx.doi.org/10.1115/1.4031470. 
Thompson, M. K., Moroni, G., Vaneker, T., Fadel, G., Campbell, R. I., Gibson, I., Bernard, A., Schulz, J., Graf, P., Ahuja, B. and Martina, F. (2016), "Design for additive manufacturing: Trends, opportunities, considerations, and constraints”, CIRP Annals, Vol. 65(2), pp. 737 - 760, https://doi.org/10.1016/j.cirp.2016.05.004.

United Nations (2015), "Transforming our world: the 2030 Agenda for sustainable development", A/RES/70/1, available at sustainabledevelopment.un.org.

Von Hippel, E. (2005), "Democratizing innovation”, The MIT press, https://doi.org/10.7551/mitpress/2333.001.0001.

\section{Author Biography}

Serena Graziosi is an Associate Professor in the Department of Mechanical Engineering of Politecnico di Milano. Her research activities are focused on the definition of design methods, tools, and processes to drive the development of new products and services; her research interests include Design for Additive Manufacturing, Experience Design, Design for Behaviour Change, and Virtual Prototyping.

Armando V. Razionale is an Associate Professor at the School of Engineering of the University of Pisa, where he received his Master's degree in Aeronautical Engineering in 2001 and a PhD degree in Mechanical Engineering in 2004. He is a member of the Council of the Master Degree in Mechanical Engineering, Management Engineer, Aerospace Engineer and of the $\mathrm{PhD}$ in Smart-industry. His research activity is focused on design methods by CAD/CAE systems, optical technologies for 3D scanning, optical methods for mechanical and biomedical design. The results of his research are published in more than 50 scientific papers on international journals and congress proceedings. 